\title{
HnRNPH1/H2, U1 snRNP, and U11 snRNP cooperate to regulate the stability of the $U 11-48 K$ pre-mRNA
}

\author{
JANNE J. TURUNEN, ${ }^{1}$ BHUPENDRA VERMA, TUULA A. NYMAN, and MIKKO J. FRILANDER ${ }^{2}$ \\ Institute of Biotechnology, PL 56, FIN-00014 University of Helsinki, Helsinki, Finland
}

\begin{abstract}
Alternative splicing (AS) is a major contributor to proteome diversity, but it also regulates gene expression by introducing premature termination codons (PTCs) that destabilize transcripts, typically via the nonsense-mediated decay (NMD) pathway. Such AS events often take place within long, conserved sequence elements, particularly in genes encoding various RNA binding proteins. AS-NMD is often activated by the protein encoded by the same gene, leading to a self-regulating feedback loop that maintains constant protein levels. However, cross-regulation between different RNA binding proteins is also common, giving rise to finely tuned regulatory networks. Recently, we described a feedback mechanism regulating two protein components of the U12-dependent spliceosome (U11-48K and U11/U12-65K) through a highly conserved sequence element. These elements contain a U11 snRNP-binding splicing enhancer (USSE), which, through the U11 snRNP, activates an upstream U2-type 3'ss, resulting in the degradation of the U11-48K mRNA by AS-NMD. Through phylogenetic analysis, we now identify a G-rich sequence element that is conserved in fishes as well as mammals. We show that this element binds hnRNPF/H proteins in vitro. Knockdown of hnRNPH1/H2 or mutations in the G-run both lead to enhanced activation of the 3'ss in vivo, suggesting that hnRNPH1/H2 proteins counteract the 3 'ss activation. Furthermore, we provide evidence that U1 binding immediately downstream from the G-run similarly counteracts the U11-mediated activation of the alternative 3 'ss. Thus, our results elucidate the mechanism in which snRNPs from both spliceosomes together with hnRNPH1/H2 proteins regulate the recognition and activation of the highly conserved alternative splice sites within the U11-48K pre-mRNA.
\end{abstract}

Keywords: U1; U11; hnRNPH; U12-dependent splicing; alternative splicing

\section{INTRODUCTION}

Alternative splicing (AS) of precursor messenger RNAs (premRNAs) is a major contributor to the expansion of proteomic diversity in multicellular eukaryotes. Indeed, it has been shown that $\sim 95 \%$ of human protein coding transcripts are spliced alternatively (Pan et al. 2008; Wang et al. 2008). Inclusion or exclusion of alternative exons is generally regulated by short sequence elements located near the splice sites. These are recognized by protein factors, which, depending on the context, can either activate or inhibit the nearby splice sites. Among these proteins, two prominent classes have been distinguished: SR proteins and hnRNP proteins. Both protein types have a wide range of inhibitory and activating functions, depending both on the sequence context and on other trans-acting factors interacting with them (for review, see Chen and Manley 2009; Nilsen and Graveley 2010). It has become increasingly evident, however, that core components of

\footnotetext{
${ }^{1}$ Present address: Department of Laboratory Medicine, Karolinska Institutet, SE 14157 Huddinge, Sweden

${ }^{2}$ Corresponding author

E-mail Mikko.Frilander@Helsinki.Fi

Article published online ahead of print. Article and publication date are at http://www.rnajournal.org/cgi/doi/10.1261/rna.036715.112.
}

the spliceosome can also regulate AS (Verbeeren et al. 2010; Saltzman et al. 2011) and that changes in the levels of these components, such as U1 snRNP, can have widespread consequences for gene expression (Saltzman et al. 2011).

In addition to increasing the number of protein coding transcripts, AS can also regulate gene expression by introducing alternative exons that cause destabilization of the transcript. Such "poison," or "nonsense," exons typically cause the introduction of premature termination codons (PTCs), which target the transcripts to the nonsense-mediated decay (NMD) pathway. Nonsense exons are particularly common in genes encoding splicing factors and other RNA binding proteins and are often embedded in longer and highly conserved regulatory sequence elements (Lareau et al. 2007; Ni et al. 2007; Yeo et al. 2007; Saltzman et al. 2008, 2011). These may contain a number of binding sites for various regulatory factors, and some of these sites are presumably recognized by the protein encoded by the same gene, promoting the inclusion of the nonsense exons. This arrangement leads to a self-regulating negative feedback loop that maintains constant protein levels. However, AS-NMD events in the transcripts of splicing factors are also subject to regulation by other splicing factors, giving rise to finely tuned regulatory networks (Änkö et al. 2012; Huelga et al. 2012). 
U12-type introns are a rare group of introns with highly distinct $5^{\prime}$ splice site $\left(5^{\prime} \mathrm{ss}\right)$ and branch point sequences that are recognized by the distinct U12-dependent spliceosome (for review, see Turunen et al. 2013). Recently, we described a feedback mechanism regulating two protein components of the U12-dependent spliceosome (U11-48K and U11/U12$65 \mathrm{~K})$ through highly conserved sequence elements of $\approx 100$ bp in length (Fig. 1; Verbeeren et al. 2010). These elements contain a duplicated U12-type $5^{\prime}$ ss termed the USSE (U11 snRNP-binding splicing enhancer), which is necessary for the feedback regulation. In this system, the U11/U12 disnRNP (or possibly U11 snRNP alone) can recognize the USSE and activate an upstream $3^{\prime}$ splice site ( $3^{\prime}$ ss), leading to formation of longer, more unstable mRNAs. In the U11$48 \mathrm{~K}$ transcript, the USSE-dependent activation of the upstream $3^{\prime}$ ss causes the inclusion of an intronic region with a PTC. Activation of an additional 5'ss only 8 nucleotides (nt) downstream from this $3^{\prime}$ ss results in an inclusion of a nonsense exon and appearance of a PTC through frameshift in the following exon (see Fig. 1). Although the exon is flanked by strong U2-type splice sites, the activation of either the $3^{\prime}$ ss or the $5^{\prime}$ ss is negligible in the absence of the USSE. Here, we have studied trans-acting factors affecting the inclusion of the nonsense exon. We find that hnRNPH1/H2 (and possibly hnRNPF) proteins and U1 snRNP promote exon skipping, mainly by inhibiting the use of the $3^{\prime}$ ss upstream of the nonsense exon. Thus, our results elucidate the mechanism of recognition and activation of the highly conserved nonsense exon within the $48 \mathrm{~K}$ pre-mRNA, with snRNPs from both spliceosomes regulating splice site selection together with hnRNPH1/H2.

\section{RESULTS}

\section{Recognition of a highly conserved poison exon by $\mathrm{hnRNPF/H}$ proteins in vitro}

Our previous study (Verbeeren et al. 2010) indicated that the nonsense exon $4 \mathrm{i}$ was included into the $48 \mathrm{~K}$ mRNA at very low levels in the absence of the USSE despite the strong splice sites surrounding it, suggesting the presence of additional sequence elements that inhibit the upstream $3^{\prime}$ ss. A notable feature in this exon is a run of four G-residues, which is among the most conserved motifs in the regulatory element of the SNRNP48 gene and is present not only in all mammalian species studied but also in fishes, which show much less conservation elsewhere upstream of the USSE (Fig. 1). Such G-runs are typical binding sites for members of the hnRNPF/H protein family (Caputi and Zahler 2001; Schaub et al. 2007), which have often been found to inhibit splicing when bound to exonic splicing silencers (ESSs) (Chen et al. 1999; Buratti et al. 2004; Xiao et al. 2009; LeFave et al. 2011; Huelga et al. 2012; Wang et al. 2012). To test whether hnRNPF/H proteins bind to the G-run in exon $4 \mathrm{i}$, we performed protein-RNA crosslinking experiments with RNA substrates containing the conserved $48 \mathrm{~K}$ element and part of the preceding intron (Verbeeren et al. 2010). To detect proteins specifically binding to the G-run, a single ${ }^{32} \mathrm{P}$-labeled phosphate was placed in

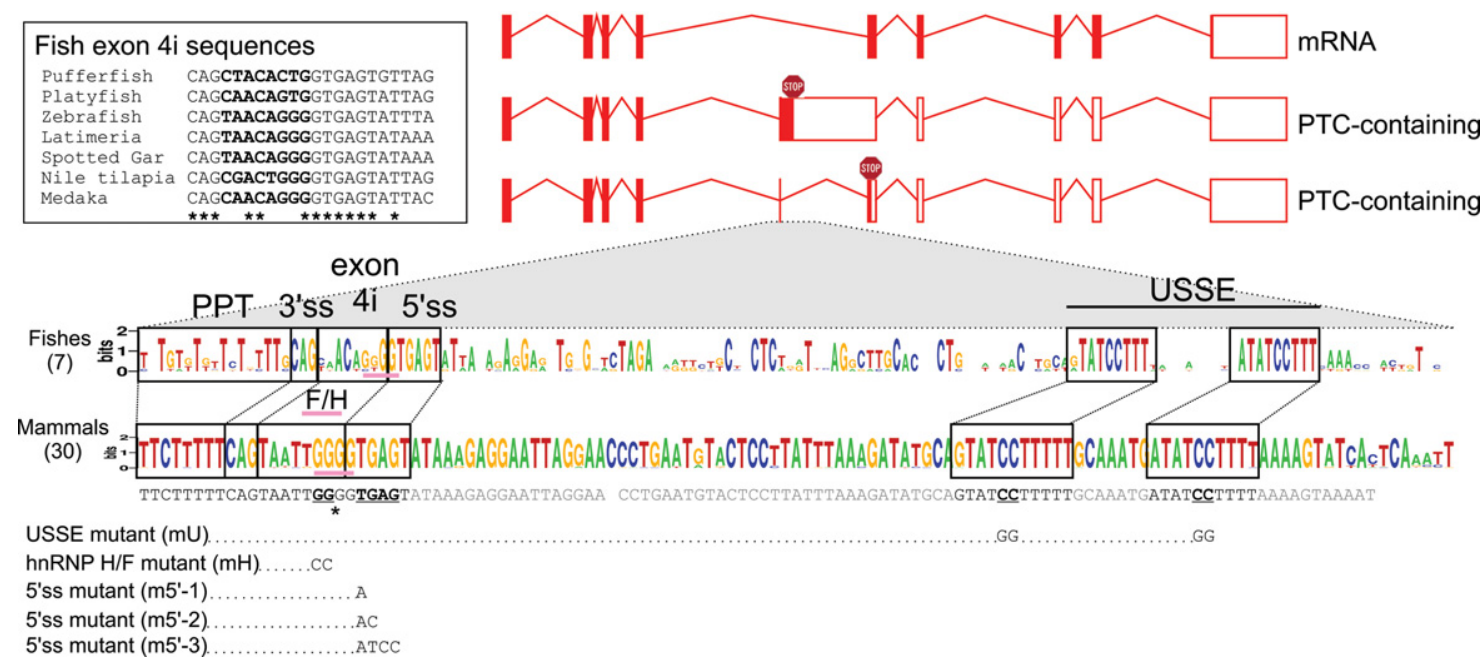

FIGURE 1. USSE and the nonsense exon within the $48 \mathrm{~K}$ pre-mRNA. Illustration of the SNRNP48 splicing patterns and the sequences within the conserved regulatory element. The exon-intron structure for the human SNRNP48 transcripts is shown schematically (top right), with the solid rectangles representing exons in the open reading frame, and open rectangles representing those in the $3^{\prime}$ UTR. The topmost splicing pattern forms the protein-coding mRNA, while the two patterns below result in PTC-containing isoforms. The conserved sequences surrounding exon $4 \mathrm{i}$ in seven individual fish species are shown in the inset, with exon $4 \mathrm{i}$ in bold and fully conserved bases indicated by asterisks (top left). The overall conservation of these sequences is analyzed in the middle panel, which shows the conserved regulatory element as a conservation diagram created with WebLogo (Crooks et al. 2004), followed by a similar analysis of 30 mammalian species (see also Verbeeren et al. 2010). The USSE and the sequence elements surrounding exon $4 \mathrm{i}$ are highlighted. Underneath the WebLogo diagram is the corresponding human sequence used in the reporter and in vitro constructs. The asterisk denotes the location of the ${ }^{32} \mathrm{P}$-labeled phosphate in the in vitro crosslinking constructs. Sites with mutations are bolded and underlined, and the corresponding mutations are shown underneath the sequence. 
the middle of it (Fig. 1). The substrates were incubated in nuclear extract and crosslinked with 254-nm UV light, followed by RNase treatment. To identify the proteins, samples were denatured to dissociate protein complexes, followed by immunoprecipitation (IP) with antibodies against a number of hnRNP and SR proteins. Proteins were then separated by SDS-PAGE and visualized by autoradiography (Fig. 2A). With the wild-type (WT) construct, two prominent crosslinks were formed (Fig. 2A, lane 1). Mutating the G-run (mH) resulted in the loss of both of these bands and the appearance of two other crosslinks with slightly differing mobilities (Fig. 2A, lane 2). Both of the crosslinks specific to the WT substrate
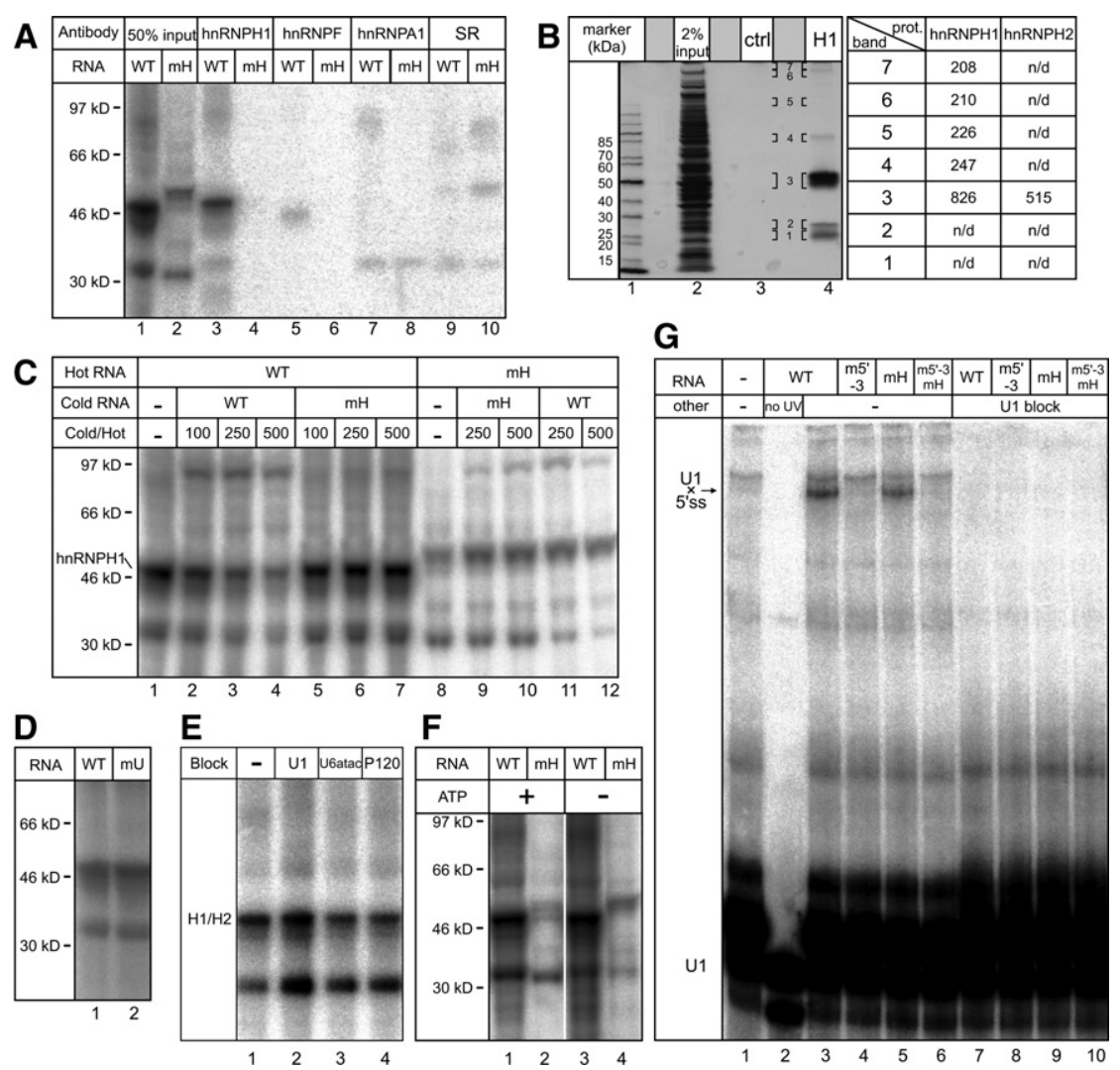

FIGURE 2. The G-rich region in exon $4 \mathrm{i}$ is recognized by hnRNP $\mathrm{F}$ and $\mathrm{H} 1 / \mathrm{H} 2$ proteins. $(A)$ Identification of protein crosslinks. Nuclear extract was incubated with WT and mutant crosslinking substrates, as indicated, and samples were crosslinked and immunoprecipitated with the indicated antibodies. Proteins were then separated by SDS-PAGE and visualized by autoradiography. Positions of marker proteins are indicated on the left. (B) IPs for mass spectrometry. Samples immunoprecipitated by hnRNPH1 antibody $(\mathrm{H} 1)$ or control beads (ctrl) were separated on a gel and silver-stained. Bands (and equivalent regions in the control lane) indicated by numbers were cut out and identified by mass spectrometry. The Mascot protein scores for the hnRNP proteins detected in each band (1-7) of the H1 IP lane are listed on the right (n/d indicates not detected). The complete database search results are available in the Supplemental Data. $(C)$ Competition assay for crosslink formation. Radioactively labeled (hot) RNA was incubated in the presence or absence of nonlabeled (cold) RNA followed by crosslinking, gel separation, and autoradiography. Cold/hot ratio indicates the amount of excess cold RNA. (D) Crosslinks were formed as in $A$ with WT or USSE mutant substrate. $(E)$ Crosslinking reactions performed in the presence of $15 \mu \mathrm{M} 2^{\prime}$-O-methyl RNA oligonucleotides against snRNAs (U1 or U6atac) or an unrelated pre-mRNA (P120). (F) Crosslinking reactions performed in the presence or absence of ATP. $(G)$ U1 snRNA crosslinks formed to the exon 4i 5'ss. Reactions performed with nonlabeled RNA were crosslinked in the presence of AMT-psoralen, and RNA was then extracted and separated by denaturing PAGE, followed by Northern blotting with a U1-specific probe. were immunoprecipitated by an hnRNPH1-specific antibody. Consistently, based on its apparent mobility $(\approx 50 \mathrm{kDa})$, the larger of the two crosslinks is in the correct size range for hnRNPH1, while the smaller band of $\approx 35 \mathrm{kDa}$ is of the correct size to be the previously characterized C-terminal fragment of hnRNPH1 or H2 (Honoré et al. 1995, 1999). HnRNPF was also detected after IP, although the crosslink is much weaker than the hnRNPH1 crosslink and could not be detected in nonimmunoprecipitated samples. However, the hnRNPF crosslink was also specific to the WT substrate and was lost when the G-run was mutated (lanes 5, 6). Other proteins deNAl and phosphorylated SR proteins. None of these were specific to the G-run, as they were also detected with the $\mathrm{mH}$ mutant (hnRNPA1: lanes 7, 8; phosphorylated SR proteins: lanes 9, 10).

HnRNPH1 and the closely related hnRNPH2 are often recognized by the same antibodies, which might also further cross-react with other members of the hnRNPF/H family (Honoré et al. 1995, 1999). Of these, hnRNPH3 has a molecular mass of $37 \mathrm{kDa}$ and therefore provides an alternative possibility for the identity of the crosslinked $35-\mathrm{kDa}$ protein. To verify the identities of the protein bands in the hnRNPH1 IP sample, we tested the specificity of the anti-H1 antibody by mass spectrometry. For this, uncrosslinked nuclear extracts were immunoprecipitated with the hnRNPH1 antibody, while empty beads were used as a control. The proteins were separated using SDS-PAGE and visualized with silver-staining. Surprisingly, no protein band corresponding to the $35-\mathrm{kDa}$ crosslink was seen on the silver-stained gels (Fig. 2B). For protein identification, the protein bands were cut out from the gel, in-gel digested into peptides, and analyzed by mass spectrometry. In addition to the immunoglobulins, only hnRNPH1 and hnRNPH2 were found to be specifically present in the anti-hnRNPH1 IPs, compared with the control samples (Fig. $2 \mathrm{~B}$; see also the Supplemental Data). The identification of trace amounts of hnRNPH1 from several bands likely results from carryover in the LC-MS/MS analysis. In conclusion, the strong bands recognized by the hnRNPH1 antibody in the crosslinking experiments are either hnRNPH1 and/or hnRNPH2. Interestingly, the absence of a clear 35-kDa band in the silver-stained gels suggests that this 
hnRNPH1/H2 fragment might be present at very low levels but could have high affinity for the G-run in exon $4 \mathrm{i}$, giving rise to the strong crosslink. Alternatively, it could be a degradation product of hnRNPH1/H2 resulting from the crosslinking/IP procedure itself.

To confirm the specificity of hnRNPH1/H2 binding, we used competition experiments with unlabeled RNAs containing either a WT or a mutated G-run. Consistently, challenging the ${ }^{32} \mathrm{P}$-labeled WT crosslinking substrate with unlabeled WT RNA, but not with mH RNA, clearly reduced hnRNPH1/H2 crosslinking efficiency in a dose-dependent manner (Fig. 2C, cf. lanes 1-4 and 5-7), suggesting sequence-specific recognition of the G-run motif. With the mutated crosslinking substrate, no bands in the size range of hnRNPH1/H2 or hnRNPF were observed. We do note, however, that a smaller $(\approx 30$ $\mathrm{kDa}$ ) crosslinked protein was efficiently competed away from the $\mathrm{mH}$ substrate by the WT competitor (lanes 8-12), indicating that this protein (possibly hnRNPA1 or an SR protein) was able to bind the mutated exon $4 \mathrm{i}$ but favored binding to the WT G-run. Together, our results show that both hnRNPH1/H2 and hnRNPF can bind to the G-run in exon $4 \mathrm{i}$, and at least hnRNPH1/H2 can bind this motif in a sequence-specific manner.

Next we asked whether hnRNPH1/H2 binding responded to U11/U12 di-snRNPs bound to the USSE or U1 bound to the $5^{\prime}$ ss downstream from the exon $4 \mathrm{i}$. Earlier we have shown that U11/U12 snRNPs bound to the USSE are necessary for exon $4 \mathrm{i}$ inclusion (Verbeeren et al. 2010), and it is possible that the USSE achieves this by inhibiting hnRNPH1/H2 binding and thereby indirectly activating the $3^{\prime}$ ss upstream of the exon 4i. However, this is not the case, as mutating the USSE (mU) did not affect hnRNPH1/H2 crosslinking efficiency (Fig. 2D), suggesting that the USSE does not affect hnRNPH1/H2 binding directly. Another possibility is that $\mathrm{U} 1$ and hnRNPH1/H2 could compete for binding due to the proximity of the 5 ss and the G-run, as has previously been observed for similarly overlapping sites (Buratti et al. 2004). In support of this possibility, blocking U1 binding with a 2'-O-methyl RNA oligo enhanced the hnRNPH1/H2 crosslink by 1.48 -fold (mean from three experiments, with a standard deviation of \pm 0.25 ) (Fig. $2 \mathrm{E}$, cf. lanes 1 and 2), and that of the $35-\mathrm{kDa}$ fragment by 2.86 -fold $( \pm 1.46)$. In contrast, control 2'-O-methyl RNA oligos of similar length (against U6atac and the $P 120$ pre-mRNA) caused no changes in hnRNPH1/H2 crosslinking. The more dramatic change seen with the $35-\mathrm{kDa}$ fragment may be due to a high specific affinity for the G-run, as discussed above. However, the 35$\mathrm{kDa}$ fragment may also comigrate with other crosslinked proteins. Thus, $\mathrm{U} 1$ may compete with other proteins in addition to hnRNPH1/H2. Some of these may be phosphorylated SR proteins (see Fig. 2A, lanes 9, 10), as a fast-migrating band decreased in intensity after ATP depletion, while the (fulllength) hnRNPH1/H2 crosslink was unaffected (Fig. 2F).

To test whether the competition between hnRNPH1/H2 and $\mathrm{U} 1$ is reciprocal, i.e., whether hnRNPH1/H2 also affects
U1 binding, we performed psoralen crosslinking with substrates containing mutations at the G-run $(\mathrm{mH})$ and/or the 5'ss ( $5^{\prime}-3$; see Fig. 1), followed by Northern blotting. As previously described (Verbeeren et al. 2010), only one prominent $\mathrm{U} 1$ crosslink was detected, and this forms specifically to the $5^{\prime} \mathrm{ss}$, as indicated by the loss of this crosslink with a $5^{\prime}$ ss mutant ( $\left.5^{\prime}-3\right)$ substrate (Fig. 2G, lane 4). In contrast, mutating the G-run had little effect on U1 crosslinking. This is surprising considering the overlapping binding sites, and we have no clear explanation for such "one-way" inhibition. However, it is possible that under the conditions used in our in vitro assay, the binding site on the substrate is nearly saturated by $\mathrm{U} 1$, while the same may not be true for hnRNPH1/H2. Taken together, our in vitro results indicate that the highly conserved G-run is recognized by hnRNPF/H proteins. Also, hnRNPH1/ $\mathrm{H} 2$ binding does not appear to be affected by the USSE in our in vitro system but may be inhibited by U1 binding to the adjacent $5^{\prime}$ ss.

\section{HnRNPH1/H2 knockdown enhances exon $4 \mathrm{i}$ activation}

Next we wanted to know whether hnRNPH1/H2 has the expected inhibitory effect on the inclusion of exon $4 \mathrm{i}$ and the downstream intronic sequences. To study this, we knocked down hnRNPH1 in HeLa cells by using increasing amounts of an siRNA that efficiently targets hnRNPH1 (Xiao et al. 2009). Efficient reduction of hnRNPH1/H2 levels by $40 \%-$ $75 \%$ (with increasing concentration of siRNA against hnRNPH1) was verified by Western blotting and hnRNPH1 mRNA levels by RT-PCR analysis (Fig. 3A), and the effect on the splicing of the $48 \mathrm{~K}$ pre-mRNA was assayed by RTqPCR (Fig. 3B-D). No reduction in the levels of hnRNPF was observed in response to hnRNPH1 knockdown (data not shown). Consistent with the inhibitory role of hnRNPH1/H2, inclusion of exon 4i is enhanced by hnRNPH1/H2 knockdown in a dose-dependent manner (Fig. 3B). This effect becomes even more prominent in the presence of cycloheximide (CHX), which blocks translation and therefore prevents the translation-dependent NMD pathway. This stabilizes the exon $4 \mathrm{i}$-containing transcripts in a manner similar to the overexpression of a dominant-negative form of the NMD factor Upf1, as previously shown by us (Verbeeren et al. 2010). Both in the presence and absence of CHX, reduced levels of RT-qPCR products are observed at the highest siRNA concentration $(50 \mathrm{nM})$, likely due to toxic effects or secondary effects caused by mis-splicing in other transcripts. Such secondary effects can be expected based on the fact that hnRNPH1regulates the splicing of other splicing factors, and its knockdown can indeed lead to changes in AS of exons that are not directly bound by it (Huelga et al. 2012).

A slight increase in the levels of the longer isoform containing both exon $4 \mathrm{i}$ and the downstream intron was also observed after knockdown. Importantly, this suggests that hnRNPH1/ $\mathrm{H} 2$ inhibition is not targeted against the $5^{\prime}$ ss, at least not 
A
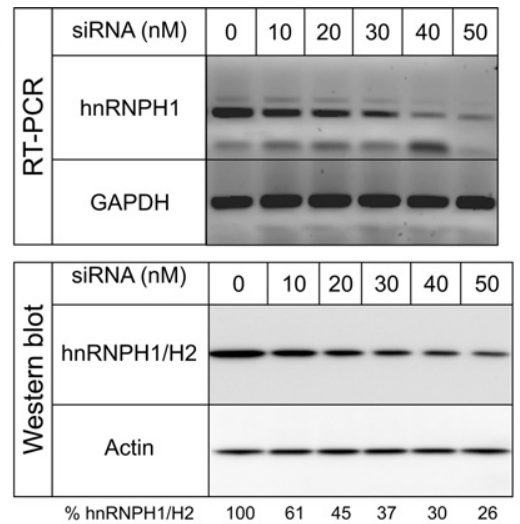

C

exon $4 i+$ intron $4 i$

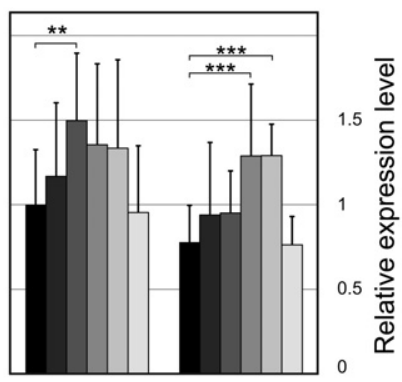

no $\mathrm{CHX}$

$\mathrm{CHX}$

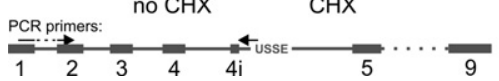

B

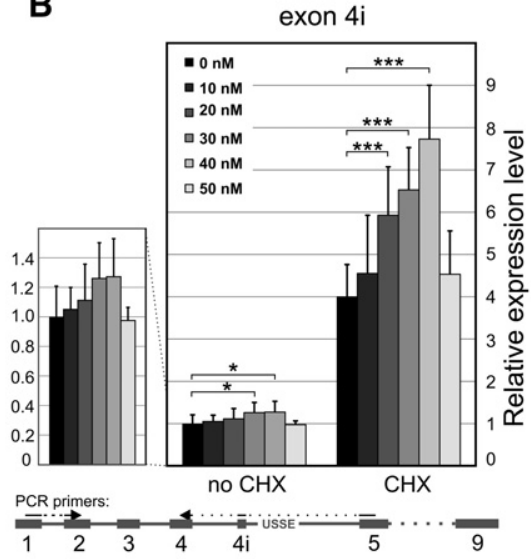

D

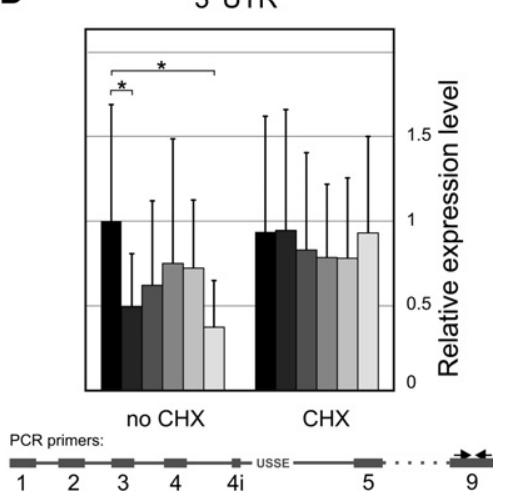

FIGURE 3. Knockdown of hnRNPH1/H2 enhances inclusion of exon 4i in vivo. HeLa cells were transfected with varying amounts of hnRNPH1 or control siRNA and in the presence or absence of cycloheximide (CHX), as indicated. (A) The concentration of hnRNPH1 (and H2) with increasing concentration of hnRNPH1 siRNA (without CHX) was analyzed by RT-PCR (H1 only) with GAPDH as a control and by Western blotting $(\mathrm{H} 1 / \mathrm{H} 2)$ with actin as a control. The fraction of hnRNPH1/H2 remaining after the knockdown is indicated (normalized against the actin signal). ( $B-D) 48 \mathrm{~K}$ mRNA isoforms were analyzed by RT-qPCR, and the values were normalized against that of the endogenous control (MARS2). For each isoform, the product level in the absence of hnRNPH1 siRNA and CHX was set as 1 . The values represent averages and standard deviations from three independent knockdown experiments, each analyzed in triplicate qPCR reactions. Knockdown samples differing significantly from the corresponding control sample in a two-tailed Student's $t$-test are marked with asterisks $\left({ }^{*} P<0.05,{ }^{* *} P<0.01,{ }^{* * *} P<0.001\right)$. The location of the qPCR primers within the $48 \mathrm{~K}$ sequence (with exons numbered) is presented schematically underneath each panel. (B) Levels of qPCR products derived from the $48 \mathrm{~K}$ isoform containing exon $4 \mathrm{i}$. The small blow-up graph displays in more detail the values for the experiments without CHX. $(C)$ Levels of qPCR products derived from the long $48 \mathrm{~K}$ isoform containing exon $4 \mathrm{i}$ and intron $4 \mathrm{i}$. (D) Levels of qPCR products derived from the $48 \mathrm{~K} 3^{\prime} \mathrm{UTR}$.

exclusively, but that the activation of the $3^{\prime}$ ss is also affected (Fig. 3C). Interestingly, unlike the isoform containing exon $4 \mathrm{i}$ alone, this isoform appears not to be stabilized by the CHX treatment, suggesting that it is not an NMD target, despite the presence of a PTC. It is possible that such transcripts are retained in the nucleus and/or degraded by processes other than NMD. In the absence of CHX, the knockdown also resulted in a slight decrease in the overall levels of the $48 \mathrm{~K}$ transcript, as measured by a qPCR assay targeting the $48 \mathrm{~K} 3^{\prime}$ UTR (Fig. 3D). This may be due to increased levels of alternatively spliced mRNAs being degraded by NMD, as no such decrease in transcript levels was observed in the presence of CHX.

\section{Inhibition of exon $4 \mathrm{i}$ inclusion by the exonic G-run and the exon $4 i 5^{\prime}$ ss in vivo}

To confirm the observed effects of the hnRNPH1/H2 knockdown, we investigated the in vivo consequences of various mutations at the hnRNPF/H binding site, at the $5^{\prime}$ ss downstream from exon $4 \mathrm{i}$ and at the USSE (see Fig. 1) using reporter constructs (see Fig. 4A) containing the highly conserved $48 \mathrm{~K}$ regulatory element (Verbeeren et al. 2010). Plasmids containing these mutations and combinations thereof were transfected into HEK293 cells, and the resulting mRNAs were analyzed by semiquantitative RTPCR. To monitor both of the alternatively spliced isoforms, i.e., the one containing exon $4 \mathrm{i}$ alone and the other containing exon $4 \mathrm{i}$ and the downstream intron, competing downstream primers were used in the RT-PCR reactions (Fig. 4A). Three 5 'ss mutants of varying severity were used in order to rule out effects related to residual U1 binding or the formation of a novel binding site for an additional splicing factor as a result of the mutation. To confirm that U1 binding was affected, the $5^{\prime}$ ss mutations were initially investigated in vitro by psoralen crosslinking, which revealed near-complete disappearance of U1-specific crosslinks with each 5 'ss mutant (Fig. 4B).

In line with our previous results (Verbeeren et al. 2010), exon $4 \mathrm{i}$ inclusion level was $\sim 33 \%$ with the WT construct, while USSE inactivation $(\mathrm{mU})$ resulted in an approximately threefold drop in exon $4 \mathrm{i}$ inclusion (Fig. 4A, cf. lanes 1 and 3). Consistent with the in vitro and the knockdown data, the mH mutant, in the presence of the WT USSE, resulted in increased inclusion of exon 4i (approximately 1.5-fold; lane 3 ), suggesting that the G-run does indeed inhibit exon $4 \mathrm{i}$ inclusion by binding hnRNPH1/H2, and possibly hnRNPF. A slight, albeit statistically insignificant increase in exon $4 \mathrm{i}$ inclusion levels was also detected when comparing the $\mathrm{mU}$ reporter with the $\mathrm{mH}-\mathrm{mU}$ double mutant (cf. lanes 2 and 4).

The constructs containing mutations in the 5 'ss revealed more dramatic changes in the splicing patterns and efficiency. Not surprisingly, the 5'ss mutations caused processing changes such that regions downstream from exon $4 \mathrm{i}$ were included, similar to the longer endogenous mRNA isoform (see Fig. 1). However, an unexpected outcome of the $5^{\prime}$ ss 


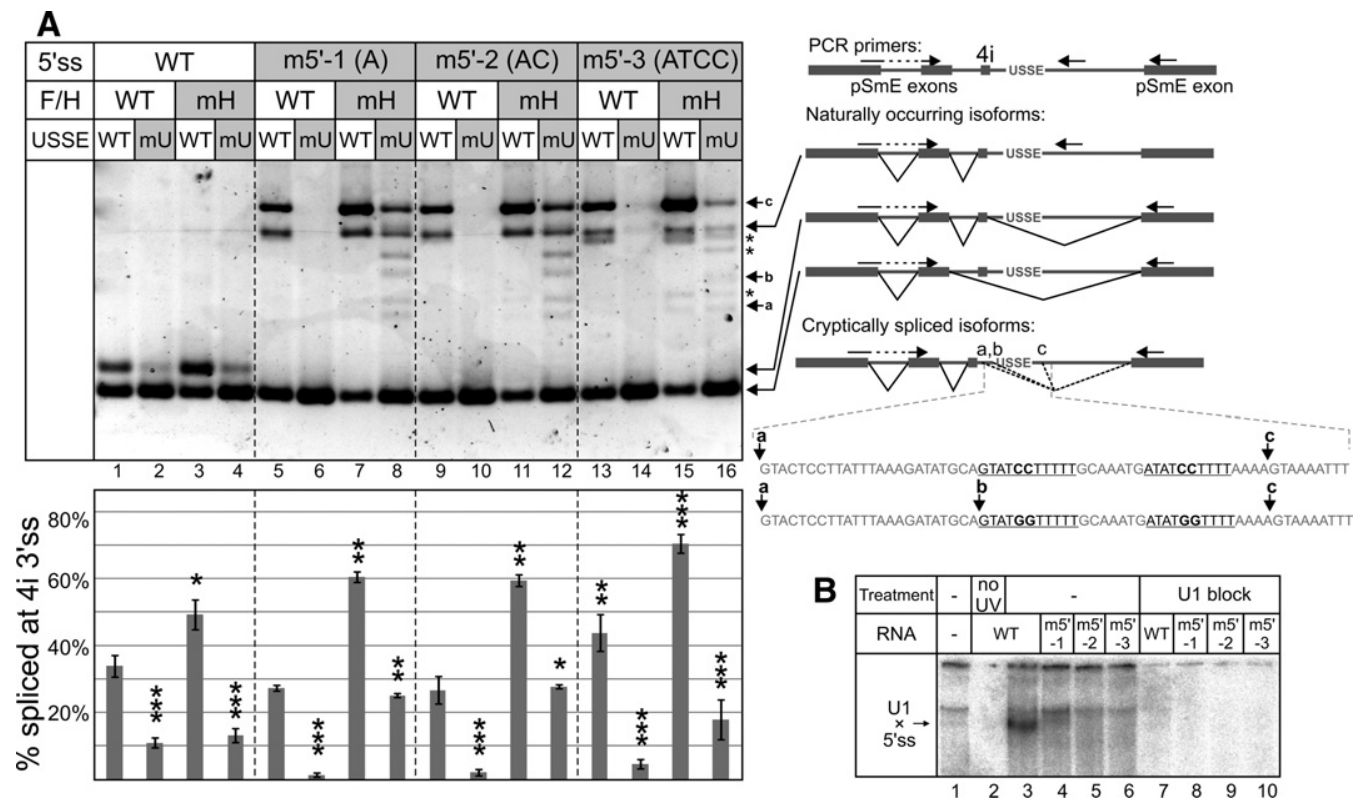

FIGURE 4. Mutations in the G-run and in the $5^{\prime}$ ss stimulate exon $4 \mathrm{i}$ inclusion. (A) Cells were transfected with WT or mutant reporter constructs as indicated above the panel. The mRNAs were analyzed by RT-PCR using three primers simultaneously. The primers and the splicing patterns are indicated schematically on the right, as are products formed by activation of cryptic 5 ' splice sites $(a-c)$. The precise positions of the detected splice sites in transcripts with or without USSE mutations (bolded; USSE underlined), as determined by sequencing, are indicated in the blow-up sequences below. Asterisks $(*)$ indicate nonreproducable cryptic products. Underneath the lanes, percentages of products spliced at the $3^{\prime}$ ss upstream of exon $4 \mathrm{i}$ are shown, with the mean and standard deviation from three independent experiments. Values differing significantly from the WT construct (lane 1) in a two-tailed Student's $t$-test are marked with asterisks $\left({ }^{*} P<0.05,{ }^{* *} P<0.01,{ }^{* * *} P<0.001\right)$. (B) Formation of U1/5'ss base pairs in $5^{\prime}$ ss mutant substrates was assayed by psoralen crosslinking as in Figure 2G.

mutations was the activation of cryptic U2-type splicing donor sites, some of which were reproducible in all repetitions of the experiment (labeled a-c in Fig. 4A). With the 5 'ss mutations alone, a splicing donor located downstream from the USSE is strongly activated (Fig. 4A variant c; lanes $5,9,13)$. When both the $5^{\prime}$ ss and G-run are mutated, there is activation of an additional, albeit weak cryptic $5^{\prime}$ ss (a) with all the $5^{\prime}$ ss mutants (lanes $7,11,15$ ). Finally, when the USSE is also mutated in this context, yet additional sites are activated (Fig. 3A, variant b; lanes 8, 12, 16), although not all of these are reproducible (Fig. 3A, asterisks). These results suggest that both the U1 and U11/U12 snRNPs may have a role in suppressing cryptic splice sites within and near the highly conserved region.

While the novel $5^{\prime}$ ss is different in each of the cryptically spliced isoforms, the exons resulting from the use of the cryptic $5^{\prime}$ ss all use the $3^{\prime}$ ss upstream of exon $4 \mathrm{i}$. It might be possible that in these isoforms, the $3^{\prime}$ ss is activated through exon definition interactions by one of the U1 snRNPs bound at the cryptic donor site. However, this appears not to be the case for the most prominent cryptic isoform (c), as inactivating the USSE together with the natural $4 \mathrm{i} 5^{\prime}$ ss resulted in almost complete inactivation of the $3^{\prime}$ ss (lanes $6,10,14$ ), even more so than with the USSE mutant alone. Thus, 3 'ss activation appears to be mediated by the USSE and not by U1 snRNP bound at the cryptic donor site $c$. We have therefore included the quantification of the cryptic isoforms in our analysis of the $3^{\prime}$ ss activation. In contrast to the $\mathrm{m}^{\prime}$-mU mutants, $5^{\prime}$ ss mutations alone did not significantly affect $3^{\prime}$ ss activation (lanes 5,9$)$, except for the most severe mutation $\left(\mathrm{m} 5^{\prime}-3\right.$, ATCC), which slightly enhances splicing (lane 13). When the $5^{\prime}$ ss mutations were combined with the G-run mutations, the $3^{\prime}$ ss activation was significantly increased (lanes 7, 11, 15). Interestingly, if the USSE is then inactivated in the presence of both the G-run and 5'ss mutations, the 3'ss usage is almost as high as with the WT reporter (lanes 8,12 ), except for the most severe $5^{\prime}$ ss mutation (lane 16), which is somewhat less active. Together these results suggest not only that hnRNPF/ $\mathrm{H}$ proteins are indeed able to bind to the G-run more efficiently in the absence of $\mathrm{U} 1$, thereby completely suppressing splicing at the $3^{\prime} \mathrm{ss}$, but that U1 binding to the $5^{\prime}$ ss can also inhibit $3^{\prime}$ ss activation.

\section{DISCUSSION}

Previously we have described a negative feedback regulation system that uses a novel splicing enhancer, USSE, embedded within a highly conserved intronic region of the SNRNP48 gene to activate AS and NMD. We showed that the short USSE element containing a duplicated U12-type $5^{\prime}$ ss is necessary for the activation of the upstream $3^{\prime}$ ss, which leads to inclusion of the NMD-inducing exon 4i (Verbeeren et al. 2010). As the USSE itself accounts for only a small portion of the conserved element, we have here investigated the 
role of other sequence motifs therein. We find that a G-run within exon $4 \mathrm{i}$, which is present in all mammalian and most fish species, is specifically recognized by proteins of the hnRNPF/H family, i.e., hnRNPF and hnRNPH1 and/or hnRNPH2 (Fig. 2). Of these, at least hnRNPH1 and/or H2 acts as negative regulator that suppresses the upstream $3^{\prime}$ ss (Figs. 2-4). The hnRNPF/H binding site overlaps with a consensus $5^{\prime}$ ss downstream from exon $4 \mathrm{i}$, which is recognized by the U1 snRNP. Our data suggest that hnRNPH1/H2 and U1 compete for binding to exon $4 \mathrm{i}$ and are each able to suppress the 3 'ss activation mediated by the USSE. Interestingly, even though our results confirm our earlier observation that U11/ U12 snRNP binding to the USSE is needed for $3^{\prime}$ ss activation, we find that when both the G-run and the $5^{\prime}$ ss are mutated, the 3 'ss is used efficiently even in the absence of a functional USSE element. This suggests that hnRNPF/H proteins and $\mathrm{U} 1$ act together to constitute a key negative regulator that counteracts the USSE activity.

Our observation that exon-bound hnRNPF/H proteins function as splicing repressors is consistent with previous reports that have documented a similar activity, as well as with large-scale increase in inclusion of G-run-containing exons after hnRNPH1 knockdown (Chen et al. 1999; Buratti et al. 2004; Xiao et al. 2009). In previous studies on regulation of alternative exons, hnRNPH1/H2 and $\mathrm{F}$ have been found to act in a redundant manner in certain contexts, while in others their functions are not interchangeable (Crawford and Patton 2006; McNally et al. 2006; Mauger et al. 2008; Coles et al. 2009; Huelga et al. 2012). Members of the hnRNPF/H protein family have also been implicated in the formation of larger complexes, in which their functions are not redundant (Schaub et al. 2007). As the crosslinking efficiency of hnRNPF compared with hnRNPH1/H2 is low (Fig. 2), it is possible that hnRNPH1 and/or $\mathrm{H} 2$ may be the most crucial of these proteins for exon $4 \mathrm{i}$ regulation. However, in the absence of data directly addressing the role of hnRNPF, we cannot conclusively show whether it also has a role in regulating exon $4 \mathrm{i}$ splicing. At present, we cannot therefore distinguish whether the hnRNPF/H proteins have specific roles in regulating exon $4 \mathrm{i}$ splicing, or whether their functions are redundant.

While our in vitro data only show inhibition of hnRNPH1/ H2 binding by U1 (Fig. 2), our in vivo data strongly suggest that $\mathrm{U} 1$ and hnRNPH1/H2 inhibit each other from binding to exon $4 \mathrm{i}$ (Fig. 4A), as previously observed for a similarly overlapping G-run and 5'ss (Buratti et al. 2004). However, our results are not consistent with a simple model, in which hnRNPH1/H2 represses and U1 activates exon inclusion. Instead, splicing to the $3^{\prime}$ ss upstream of exon $4 \mathrm{i}$ occurs most efficiently when both the G-run and 5'ss are mutated, which suggests that both hnRNPH1/H2 and U1 binding can suppress the $3^{\prime}$ ss (Fig. 4A). Presumably, U1 snRNP bound at the $5^{\prime}$ ss can sterically interfere with the recognition of the $3^{\prime}$ ss, which is situated only $8 \mathrm{nt}$ upstream. A very similar case has been found in the ATM pre-mRNA, where bind- ing of $\mathrm{U} 1$ to a regulatory element inhibits the activation of a 3 'ss situated 12 nt upstream, apparently by promoting unproductive binding of U2 snRNP (Lewandowska et al. 2005; Dhir et al. 2010).

It may appear contradictory that U1 snRNP should inhibit splicing of exon $4 \mathrm{i}$ at the $3^{\prime}$ ss, while in the endogenous transcripts and the WT reporters, it very clearly also functions in defining the $5^{\prime}$ ss for removal of the downstream intron (Fig. 4). To resolve this apparent contradiction, we propose that these two functions take place at different stages: With short exons such as exon 4i, U1 is unable to contribute to exon definition interactions (Sterner and Berget 1993; Hwang and Cohen 1997; Lewandowska et al. 2005) and is instead likely to inhibit splicing by steric interference. However, following the activation of the 3 'ss by the USSE and removal of the upstream intron, U1 snRNP can then function in defining the $5^{\prime}$ ss of the downstream intron. Such a strict preferential order for upstream versus downstream intron removal was also observed for the alternative exon in the ATM transcript (Lewandowska et al. 2005). Thus, the 3'ss appears to be the primary target of splicing repression and activation. Of the two elements negatively regulating the $3^{\prime}$ ss activation, the G-run appears to be a more dominant repressor as it can completely block splicing to the exon $4 \mathrm{i} 3^{\prime}$ ss in the absence of the U2-type $5^{\prime}$ ss and the USSE. In contrast, reporters with mutations at the G-run and USSE, but with a functional 5'ss, still support exon $4 \mathrm{i}$ inclusion at low levels (Fig. 4A).

Our data indicate that while both of the alternatively spliced $48 \mathrm{~K}$ isoforms include PTCs, only the isoform containing exon $4 \mathrm{i}$ alone is degraded by NMD (Fig. 3). We do not currently know if the longer isoform containing the downstream intron is specifically degraded by another mechanism in either the nucleus or the cytoplasm. As it is present only as a minor fraction under normal circumstances (Fig. 4; data not shown), it seems likely that after exons 4 and $4 \mathrm{i}$ are ligated, most of the $48 \mathrm{~K}$ transcripts are further spliced to join exons $4 \mathrm{i}$ and 5 . In this scenario, AS-NMD-mediated feedback regulation requires an end-product containing exon $4 \mathrm{i}$ alone, with the longer isoform serving only as an intermediate (see Fig. 5). However, further analyses will be required to verify the specific roles of the two alternatively spliced isoforms.

Finally we note that both U1 and U11/U12 di-snRNPs may here have an additional role in suppressing cryptic U2-type $5^{\prime}$ ss activation as suggested by strong activation of the cryptic sites upon mutation of both the 5 'ss and USSE sequences (Fig. $4 \mathrm{~A})$. Consistently, U1 binding sites were found to function as potent suppressors of pseudoexon splice sites in a screen of splicing silencers (Wang et al. 2004). Similarly, U11 has also been shown to be able to inhibit U1 binding to a viral splicing regulatory element (Hibbert et al. 1999; McNally and McNally 1999), and a U12-type 5'ss been shown to suppress splice site activation when artificially placed in a pseudoexon (Dhir et al. 2010). However, it remains unclear whether the suppression 


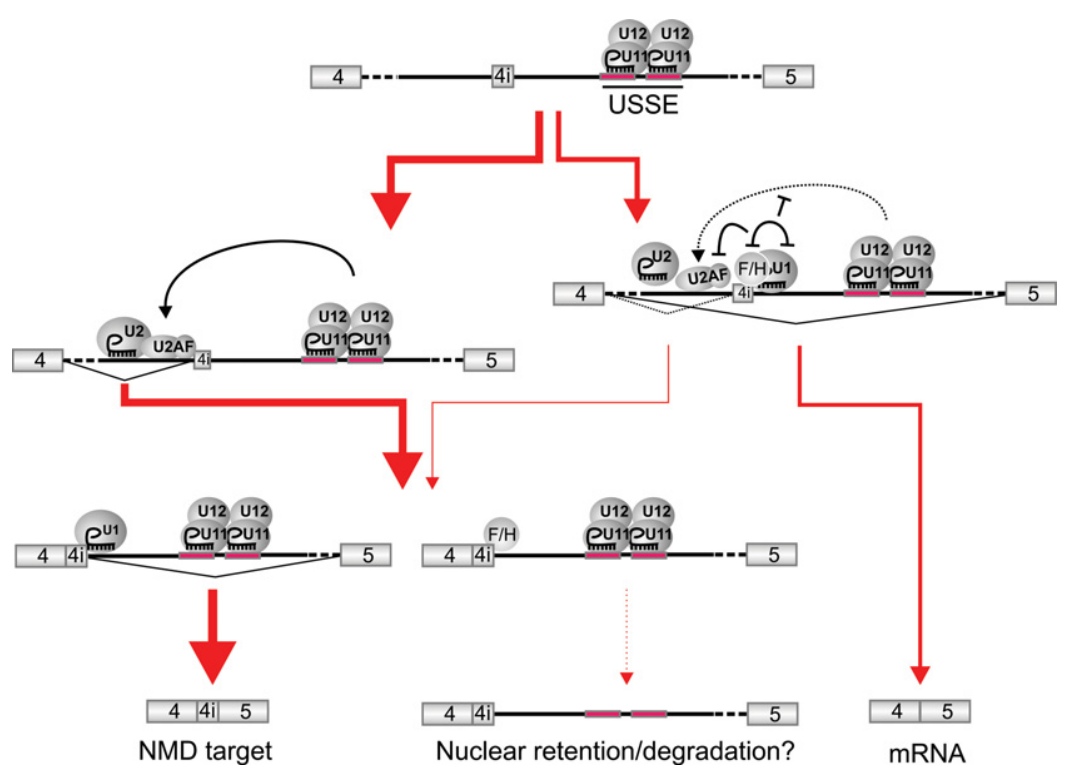

FIGURE 5. Regulation of the stability of the U11-48K mRNA. The model depicts the formation of complexes that inhibit or activate the inclusion of exon $4 \mathrm{i}$, resulting in mature mRNAs or unstable isoforms, respectively. The widths of the red arrows reflect the estimated fraction of complexes or transcripts resulting from each step (deduced from Verbeeren et al. 2010; data not shown). On the left side of the schematic, binding of U11/U12 (or U11) snRNPs to the USSE enhances binding of U2-type factors upstream of exon 4i. After exons 4 and $4 \mathrm{i}$ have been ligated, $\mathrm{U} 1$ snRNP may bind to the $5^{\prime}$ ss of exon 4 i, thus enabling the joining of exons $4 \mathrm{i}$ and 5 , although some transcripts do retain the intron downstream from exon $4 \mathrm{i}$. The inclusion of exon $4 \mathrm{i}$ is inhibited by the binding of hnRNPF/H proteins or the (premature) binding of U1 snRNP (right side of the schematic), although these factors also compete with each other. Inhibition may be caused by direct blockage of the $3^{\prime}$ ss or by blocking the interactions that mediate the enhancing effect of the USSE. Presumably, most of the transcripts with the inhibitory complex are then spliced to exclude exon $4 \mathrm{i}$, thus maturing into functional mRNAs. in bone marrow CD34-positive cells and B lymphoblast, as are hnRNPs F and $\mathrm{H} 1$ (Yeo et al. 2007; Ke and Chasin 2010). In addition to the conserved motifs characterized in this study, the region between exon $4 \mathrm{i}$ and USSE as a whole is almost $100 \%$ conserved in mammalian species. This suggests that the sequences within this region have additional significance for regulating the inclusion of exon 4i. One interesting possibility is that the sequences may be involved in secondary structures that affect splice site choice. However, if present, such structures would likely be restricted to mammals, as the sequences outside the USSE and exon $4 \mathrm{i}$ are poorly conserved in fishes. Another, perhaps more likely, function involves the binding of additional splicing factors. Several recent studies suggest that cross-regulation by multiple splicing factors is a common mechanism for regulating AS-NMD in splicing factor transcripts (Dreumont et al. 2010; Änkö et al. 2012; Huelga et al. 2012). It is likely that the conserved region within the $U 11-48 \mathrm{~K}$ transcript similarly provides yet additional binding sites for other regulatory factors, such as SR proteins and other hnRNP proteins. of cryptic splice sites has any biological function in the $48 \mathrm{~K}$ pre-mRNA, particularly as natural mRNA isoforms spliced at these sites have not been observed, and we also did not detect such endogenous transcripts after the hnRNPH1/H2 knockdown (data not shown). Within the context of our reporter constructs, U1 snRNPs bound at the cryptic sites may mediate exon definition interactions to enhance the activation of the 3'ss, which is located 41-96 nt upstream of the cryptic sites. However, such interactions do not appear to be prominent, as $3^{\prime}$ ss activation is dependent on a functional USSE while the hnRNPF/H binding site is intact. It seems likely that, as with the bona fide $5^{\prime}$ ss, activation of the $3^{\prime}$ ss upstream of exon $4 \mathrm{i}$ takes place first, followed later by splicing at one of the cryptic 5'ss.

Together our results provide evidence that the U11-48K protein feedback regulation is controlled not only by the USSE and U11/U12 di-snRNP but also by hnRNPH1/H2 and the U1 snRNP. A model including these components is presented in Figure 5. The additional components in the feedback circuit may provide opportunities for fine-tuning the regulation, possibly in a tissue-specific manner as described recently for hnRNPF/H proteins (LeFave et al. 2011). In this regard, it is interesting to note that U12-type intron-containing genes are indeed differentially expressed

\section{MATERIALS AND METHODS}

\section{Plasmids, oligonucleotides, and antibodies}

The reporter plasmid pSmE-48KUSSE contains exon $4 \mathrm{i}$ and flanking intronic sequences of the SNRNP48 gene cloned between exons of the pSmE construct (see also Fig. 4A; Verbeeren et al. 2010). The mutated reporter constructs were made by PCR and confirmed by sequencing. Primers used in RT-PCR analysis were the vector-specific primers SmE-1 CAGCCCATCAACCTTATCTT and SmE-2 GGAAGCTCCAATAATACAACCT, and the 48K-specific primer h48K-147 CAATTCAAGACTATAATATGTGACAGG. RNA oligonucleotides for the construction of in vitro crosslinking substrates were ordered from Ambion and were AAUGCAGUUUGUUG CUUAACCUUUCCUGUACUUUUUCUUUUUCAGUAAUUGG for the WT and the same with the last 2 nt replaced by CC for the $\mathrm{mH}$ mutant. The 2'-O-methyl RNA oligonucleotides against U6atac snRNA and P120 pre-mRNA had the sequences UGCUAA CCUUCU and CCUGCAAGGAUAUCCUG, respectively. The oligonucleotide $\mathrm{U}_{1-14}$ used for blocking U1 snRNA was described by Tarn and Steitz (1994). The siRNA against hnRNPH1/H2 was as described previously (Xiao et al. 2009), and a control siRNA (siGenome nontargeting siRNA, D-001210-02) was obtained from Dharmacon. Quantitative PCR was performed with the following primer pairs, with their targets given in parentheses: CCTTCGA TTACTTTGAATAAGGAC and AATGAAGAATAAATTCCCCAA 
TTA (48K exon 4i), GACTCACAATTCCAGATAA and TCAGG TTCCTAATTCCTC (48K exon $4 \mathrm{i}$ and intron $4 \mathrm{i}$ ), GGCTTCAGG TATGGGAAACAGC and TCACTCCCAAGTCCTTGCTTTT (48K 3' UTR), and CTACCTGCTTCCCTAGTG and ATCCGAAAG TTATCATAGTGG (MARS2). Antibodies used in this study were against hnRNPA1 (Abnova, clone 9H10), hnRNPF (Millipore, clone 3H4), hnRNPH1 (Acris Antibodies, catalog no. AP19044PU-N), phospho-SR proteins (Invitrogen, clone 1H4), and actin (Santa Cruz Biotechnology AC-15, catalog no. sc-69879).

\section{Sequence analyses}

The sequence comparisons of the conserved intronic element of vertebrate orthologs of the human SNRNP48 gene were performed according to the method described previously (Verbeeren et al. 2010), supplemented with additional SNRNP48 ortholog sequences obtained from www.ensembl.org. The following animal sequences were added to those previously analyzed (Ensembl gene accession numbers shown): gibbon (Nomascus leucogenys; ENSNLEG000000 11667), gorilla (Gorilla gorilla; ENSGGOG00000013220), marmoset (Callithrix jacchus; ENSCJAG00000021330), panda (Ailuropoda melanoleuca; ENSAMEG00000017349), rabbit (Oryctolagus cuniculus; ENSOCUG00000005168), and tarsier (Tarsius syrichta; ENSTSYG 00000000698). The Nile tilapia (Oreochromis niloticus) sequence was obtained from BouillaBase.org (http:// cichlid.umd.edu/ cichlidlabs/kocherlab/bouillabase.html; sequence identifier Locus_ 15407_Transcript_1/1_Confidence_1.000). 48K sequences for Spotted gar (Lepisosteus oculatus) and Platyfish (Xiphophorus maculatus) were obtained from pre.ensemb.org from chromosome LG11:10423900-10431699 and scaffold JH556728.1: 21751962178135 , respectively.

\section{In vitro RNA constructs and crosslinking}

Nonlabeled RNA substrates for in vitro reactions were prepared according to the method previously described (Verbeeren et al. 2010). Site-specifically labeled RNAs with similar sequences were constructed essentially according to the method previously described by Turunen et al. (2008). Briefly, the $3^{\prime}$ portion of the construct (starting with the last nucleotide of exon 4i) was produced by in vitro transcription with T7 RNA polymerase, dephoshorylated, and labeled at the $5^{\prime}$ end with $\left[{ }^{32} \mathrm{P}\right]$-phosphate. This fragment was then ligated to WT or mutant RNA oligonucleotides using T4 DNA ligase. Reactions were prepared, and psoralen crosslinking and Northern blotting were performed as previously described (Verbeeren et al. 2010). Site-specific protein-RNA crosslinks were formed by irradiating samples with 254-nm UV light $\left(1 \mathrm{~J} / \mathrm{cm}^{2}\right)$ on ice. For IP, samples were denatured with $0.2 \%$ SDS at $95^{\circ} \mathrm{C}$, incubated with antibodies, and washed according to the method described by Turunen et al. (2008). Proteins were separated by SDS-PAGE and visualized by autoradiography on phosphoimager plates, which were scanned with Fuji FLA-5000 scanner, and the obtained images were quantified using AIDA image analyzer software.

\section{Mass spectrometry}

Nuclear extracts were immunoprecipitated with hnRNPH1 antibody or with empty beads as a control. The proteins were separated using SDS-PAGE and visualized with silver-staining (O'Connell and Stults 1997). For protein identification, the protein bands were cut out from the gel. Proteins were in-gel digested with trypsin, and the resulting peptides were analyzed by liquid chromatography tandem mass spectrometry (LC-MS/MS) using an Ultimate 3000 nano-LC (Dionex) and a QSTAR Elite hybrid quadrupole TOFMS (Applied Biosystems / MDS Sciex) with nano-ESI ionization according to the method described previously (Öhman et al. 2010). The LC-MS/MS data was searched with in-house Mascot version 2.2 through ProteinPilot 3.0 interface against the SwissProt 2011 database. The search criteria for Mascot searches were as follows: trypsin digestion with one missed cleavage allowed, carbamidomethyl modification of cysteine as a fixed modification, and oxidation of methionine as a variable modification. Both human-specific and taxon nonspecific searches were performed (see the Supplemental Data). All of the reported protein identifications are statistically significant $(P<0.05)$.

\section{RNAi and qPCR}

For hnRNPH1 knockdown, HeLa cells were transfected with siRNAs using Lipofectamine RNAimax (Invitrogen) according to the manufacturer's instructions. Cells were transfected with varying amounts of control and hnRNPH1 siRNA, with a total siRNA concentration of $50 \mathrm{nM}$. After $48 \mathrm{~h}$, total RNA was extracted from the cells, and cDNA was synthesized with random primers and RevertAid Premium reverse transcriptase (Fermentas). A subset of knockdowns were treated with $25 \mu \mathrm{g} / \mathrm{mL}$ CHX for $2 \mathrm{~h}$ prior to RNA extraction. Real-time qPCR was performed with the Roche LightCycler 480 system, using the LightCycler 480 SYBR Green I Master mix. Absolute quantification of the products was performed using the fit points method, and the concentration of $48 \mathrm{~K}$ isoforms was further normalized against that of the endogenous control (MARS2).

\section{Reporter experiments}

HEK293 cells were grown and transfected, and total RNA extracted and RT-PCR were performed according to the method described by Verbeeren et al. (2010). The identity of the RT-PCR products was verified by sequencing. The Agarose gels were scanned with Fuji LAS-3000 cooled CCD camera, and the obtained images were quantified using AIDA image analyzer software. Intensities were normalized according to the lengths of the PCR products.

\section{SUPPLEMENTAL MATERIAL}

Supplemental material is available for this article.

\section{ACKNOWLEDGMENTS}

We thank Marja-Leena Peltonen for excellent technical assistance. This work was supported by grants from Academy of Finland, Sigrid Jusélius Foundation, and Biocentrum Helsinki to M.J.F. J.J.T. was supported by the Helsinki Graduate Program in Biotechnology and Molecular Biology.

Received October 5, 2012; accepted December 13, 2012. 


\section{REFERENCES}

Änkö ML, Müller-McNicoll M, Brandl H, Curk T, Gorup C, Henry I, Ule J, Neugebauer KM. 2012. The RNA-binding landscapes of two SR proteins reveal unique functions and binding to diverse RNA classes. Genome Biol 13: R17.

Buratti E, Baralle M, De Conti L, Baralle D, Romano M, Ayala YM, Baralle FE. 2004. hnRNP H binding at the $5^{\prime}$ splice site correlates with the pathological effect of two intronic mutations in the NF-1 and TSH $\beta$ genes. Nucleic Acids Res 32: 4224-4236.

Caputi M, Zahler AM. 2001. Determination of the RNA binding specificity of the heterogeneous nuclear ribonucleoprotein (hnRNP) H/H'/F/2H9 family. J Biol Chem 276: 43850-43859.

Chen M, Manley JL. 2009. Mechanisms of alternative splicing regulation: Insights from molecular and genomics approaches. Nat Rev Mol Cell Biol 10: 741-754.

Chen CD, Kobayashi R, Helfman DM. 1999. Binding of hnRNP H to an exonic splicing silencer is involved in the regulation of alternative splicing of the rat $\beta$-tropomyosin gene. Genes Dev 13: 593-606.

Coles JL, Hallegger M, Smith CW. 2009. A nonsense exon in the Tpm1 gene is silenced by hnRNP H and F. RNA 15: 33-43.

Crawford JB, Patton JG. 2006. Activation of $\alpha$-tropomyosin exon 2 is regulated by the SR protein $9 \mathrm{G} 8$ and heterogeneous nuclear ribonucleoproteins H and F. Mol Cell Biol 26: 8791-8802.

Crooks GE, Hon G, Chandonia JM, Brenner SE. 2004. WebLogo: A sequence logo generator. Genome Res 14: 1188-1190.

Dhir A, Buratti E, van Santen MA, Lührmann R, Baralle FE. 2010. The intronic splicing code: Multiple factors involved in ATM pseudoexon definition. EMBO J 29: 749-760.

Dreumont N, Hardy S, Behm-Ansmant I, Kister L, Branlant C, Stévenin J, Bourgeois CF. 2010. Antagonistic factors control the unproductive splicing of SC35 terminal intron. Nucleic Acids Res 38: $1353-1366$.

Hibbert CS, Gontarek RR, Beemon KL. 1999. The role of overlapping $\mathrm{U} 1$ and $\mathrm{U} 115^{\prime}$ splice site sequences in a negative regulator of splicing. RNA 5: 333-343.

Honoré B, Rasmussen HH, Vorum H, Dejgaard K, Liu X, Gromov P, Madsen P, Gesser B, Tommerup N, Celis JE. 1995. Heterogeneous nuclear ribonucleoproteins $\mathrm{H}, \mathrm{H}^{\prime}$, and $\mathrm{F}$ are members of a ubiquitously expressed subfamily of related but distinct proteins encoded by genes mapping to different chromosomes. J Biol Chem 270: 28780-28789.

Honoré B, Vorum H, Baandrup U. 1999. hnRNPs H, $\mathrm{H}^{\prime}$ and $\mathrm{F}$ behave differently with respect to posttranslational cleavage and subcellular localization. FEBS Lett 456: 274-280.

Huelga SC, Vu AQ, Arnold JD, Liang TY, Liu PP, Yan BY, Donohue JP, Shiue L, Hoon S, Brenner S, et al. 2012. Integrative genome-wide analysis reveals cooperative regulation of alternative splicing by hnRNP proteins. Cell Rep 1: 167-178.

Hwang DY, Cohen JB. 1997. U1 small nuclear RNA-promoted exon selection requires a minimal distance between the position of $\mathrm{U} 1$ binding and the 3' splice site across the exon. Mol Cell Biol 17: 7099-7107.

$\mathrm{Ke}$ S, Chasin LA. 2010. Intronic motif pairs cooperate across exons to promote pre-mRNA splicing. Genome Biol 11: R84.

Lareau LF, Inada M, Green RE, Wengrod JC, Brenner SE. 2007. Unproductive splicing of SR genes associated with highly conserved and ultraconserved DNA elements. Nature 446: 926-929.

LeFave CV, Squatrito M, Vorlova S, Rocco GL, Brennan CW, Holland EC, Pan Y-X, Cartegni L. 2011. Splicing factor hnRNPH drives an oncogenic splicing switch in gliomas. $E M B O J$ 30: 4084-4097.

Lewandowska MA, Stuani C, Parvizpur A, Baralle FE, Pagani F. 2005. Functional studies on the ATM intronic splicing processing element. Nucleic Acids Res 33: 4007-4015.

Mauger DM, Lin C, Garcia-Blanco MA. 2008. hnRNP H and hnRNP F complex with Fox2 to silence fibroblast growth factor receptor 2 exon IIIc. Mol Cell Biol 28: 5403-5419.
McNally LM, McNally MT. 1999. U1 small nuclear ribonucleoprotein and splicing inhibition by the rous sarcoma virus negative regulator of splicing element. J Virol 73: 2385-2393.

McNally LM, Yee L, McNally MT. 2006. Heterogeneous nuclear ribonucleoprotein $\mathrm{H}$ is required for optimal U11 small nuclear ribonucleoprotein binding to a retroviral RNA-processing control element: Implications for U12-dependent RNA splicing. J Biol Chem 281: 2478-2488.

Ni JZ, Grate L, Donohue JP, Preston C, Nobida N, O’Brien G, Shiue L, Clark TA, Blume JE, Ares M Jr. 2007. Ultraconserved elements are associated with homeostatic control of splicing regulators by alternative splicing and nonsense-mediated decay. Genes Dev 21: 708-718.

Nilsen TW, Graveley BR. 2010. Expansion of the eukaryotic proteome by alternative splicing. Nature 463: 457-463.

O'Connell KL, Stults JT. 1997. Identification of mouse liver proteins on two-dimensional electrophoresis gels by matrix-assisted laser desorption/ionization mass spectrometry of in situ enzymatic digests. Electrophoresis 18: 349-359.

Öhman T, Lietzén N, Välimäki E, Melchjorsen J, Matikainen S, Nyman TA. 2010. Cytosolic RNA recognition pathway activates 14-3-3 protein mediated signaling and caspase-dependent disruption of cytokeratin network in human keratinocytes. J Proteome Res 9: 1549-1564.

Pan Q, Shai O, Lee LJ, Frey BJ, Blencowe BJ. 2008. Deep surveying of alternative splicing complexity in the human transcriptome by high-throughput sequencing. Nat Genet 40: 1413-1415.

Saltzman AL, Kim YK, Pan Q, Fagnani MM, Maquat LE, Blencowe BJ. 2008. Regulation of multiple core spliceosomal proteins by alternative splicing-coupled nonsense-mediated mRNA decay. Mol Cell Biol 28: $4320-4330$

Saltzman AL, Pan Q, Blencowe BJ. 2011. Regulation of alternative splicing by the core spliceosomal machinery. Genes Dev 25: 373-384

Schaub MC, Lopez SR, Caputi M. 2007. Members of the heterogeneous nuclear ribonucleoprotein $\mathrm{H}$ family activate splicing of an HIV-1 splicing substrate by promoting formation of ATP-dependent spliceosomal complexes. J Biol Chem 282: 13617-13626.

Sterner DA, Berget SM. 1993. In vivo recognition of a vertebrate mini-exon as an exon-intron-exon unit. Mol Cell Biol 13: 26772687.

Tarn WY, Steitz JA. 1994. SR proteins can compensate for the loss of U1 snRNP functions in vitro. Genes Dev 8: 2704-2717.

Turunen JJ, Will CL, Grote M, Lührmann R, Frilander MJ. 2008. The U11-48K protein contacts the $5^{\prime}$ splice site of U12-type introns and the U11-59K protein. Mol Cell Biol 28: 3548-3560.

Turunen JJ, Niemelä EH, Verma B, Frilander MJ. 2013. The significant other: Splicing by the minor spliceosome. Wiley Interdiscip Rev RNA 4: 61-76.

Wang Z, Rolish ME, Yeo G, Tung V, Mawson M, Burge CB. 2004. Systematic identification and analysis of exonic splicing silencers. Cell 119: 831-845.

Wang ET, Sandberg R, Luo S, Khrebtukova I, Zhang L, Mayr C, Kingsmore SF, Schroth GP, Burge CB. 2008. Alternative isoform regulation in human tissue transcriptomes. Nature 456: 470-476.

Wang Y, Meng M, Xiao X, Wang Z. 2012. Intronic splicing enhancers, cognate splicing factors and context-dependent regulation rules. Nat Struct Mol Biol 19: 1044-1052.

Verbeeren J, Niemelä EH, Turunen JJ, Will CL, Ravantti JJ, Lührmann R, Frilander MJ. 2010. An ancient mechanism for splicing control: U11 snRNP as an activator of alternative splicing. Mol Cell 37: 821-833.

Xiao X, Wang Z, Jang M, Nutiu R, Wang ET, Burge CB. 2009. Splice site strength-dependent activity and genetic buffering by poly- $G$ runs. Nat Struct Mol Biol 16: 1094-1100.

Yeo GW, Van Nostrand EL, Liang TY. 2007. Discovery and analysis of evolutionarily conserved intronic splicing regulatory elements. PLoS Genet 3: e85. 

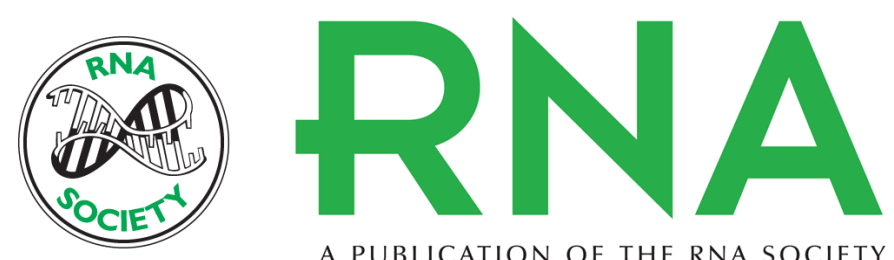

A PUBLICATION OF THE RNA SOCIETY

\section{HnRNPH1/H2, U1 snRNP, and U11 snRNP cooperate to regulate the stability of the U11-48K pre-mRNA}

Janne J. Turunen, Bhupendra Verma, Tuula A. Nyman, et al.

RNA 2013 19: 380-389 originally published online January 18, 2013

Access the most recent version at doi:10.1261/rna.036715.112

\section{Supplemental http://rnajournal.cshlp.org/content/suppl/2013/01/09/rna.036715.112.DC1 \\ Material}

References This article cites 40 articles, 18 of which can be accessed free at:

http://rnajournal.cshlp.org/content/19/3/380.full.html\#ref-list-1

\section{License}

Email Alerting Receive free email alerts when new articles cite this article - sign up in the box at the Service top right corner of the article or click here.

To subscribe to RNA go to:

http://rnajournal.cshlp.org/subscriptions 